\title{
Analytical Discrete-Time Models of Insect Population Dynamics
}

\author{
Abhyudai Singh \\ University of Delaware, Newark, DE 19716 \\ absingh@udel.edu
}

Insect population dynamics has been extensively studied using two different approaches: continuous-time and discrete-time models. The continuous-time framework is generally used to model populations with overlapping generations and all year-round reproduction. In contrast, discrete-time models are more suited for populations with non-overlapping generations that reproduce in a discrete pulse determined by season. We revisit simple models of host-parasitoid interactions and describe tools for elucidating their dynamical behaviors. One advantage of simple models is that they are often analytically tractable providing key insights into regulatory mechanisms and parameter regions that lead to stable, unstable or oscillatory population dynamics. While reviewing classical models introduced decades ago, we also highlight new modeling frameworks and results from recent literature.

\section{Simple Discrete-Time Models}

Discrete-time models have been a tradition in arthropod host-parasitoid systems and their usage is primarily motivated by the univoltine life histories of insects residing in the temperate regions of the world [1]. A typical life cycle consists of adult hosts emerging during spring, laying eggs that hatch into larvae. Hosts then overwinter in the pupal stage, and metamorphosize as adults the following year. The host becomes vulnerable to parasitoid attacks at one stage of its life cycle (typically the larval stage). Adult female parasitoids search and attack hosts during this time window of vulnerability. While adult parasitoids die after this time window, the parasitized host support juvenile parasitoids, which pupate, overwinter, and emerge as adult parasitoids the following year. Synchronized life cycles, with no overlap of generations in both the host and the parasitoid makes discrete-time models highly appropriate for these systems. 
A model describing host-parasitoid dynamics in discrete-time is given by

$$
\begin{aligned}
H_{t+1} & =R H_{t} f\left(H_{t}, P_{t}\right) \\
P_{t+1} & =k R H_{t}\left[1-f\left(H_{t}, P_{t}\right)\right]
\end{aligned}
$$

where $H_{t}$ and $P_{t}$ are the adult host and the adult parasitoid densities, respectively, at the start of year $t$, and $R>1$ denotes the host's reproduction rate. If the host is vulnerable to the parasitoid at its larval stage, then $R H_{t}$ is the host larval density exposed to parasitoid attacks. The function $f\left(H_{t}, P_{t}\right)$ is the fraction of host larvae escaping parasitism and has been referred to in literature as the escape response. In the absence of the parasitoid $f\left(H_{t}, 0\right)=1$, and the host population grows unboundedly as $H_{t+1}=R H_{t}$. Finally, $R H_{t}\left[1-f\left(H_{t}, P_{t}\right)\right]$ is the net density of parasitized larvae, with each larva giving rise to $k$ adult female parasitoids in the next generation. Note that a change in state variables, where now $H_{t}$ denotes the host larvae density, results in (1) being rewritten as

$$
\begin{aligned}
H_{t+1} & =R H_{t} f\left(H_{t}, P_{t}\right) \\
P_{t+1} & =k H_{t}\left[1-f\left(H_{t}, P_{t}\right)\right]
\end{aligned}
$$

$[2,3]$. While both forms of the model have appeared in literature, without loss of any generality we will focus our analysis on (1).

The simplest formulation of (1) is the classical Nicholson-Bailey model

$$
\begin{aligned}
H_{t+1} & =R H_{t} \exp \left(-c T P_{t}\right) \\
P_{t+1} & =k R H_{t}\left[1-\exp \left(-c T P_{t}\right)\right]
\end{aligned}
$$

where $c>0$ represents the rate at which parasitoids attack hosts, and $T$ is the duration of the host vulnerable stage [4]. The model assumes that parasitoids search for hosts randomly, are never egg-limited, and have quick handling times. If the number of parasitoid attacks per host follows a Poisson distribution with mean $c T P_{t}$, then the escape response $f\left(H_{t}, P_{t}\right)=\exp \left(-c T P_{t}\right)$ is the probability of zero attacks in the Poisson distribution. A typical time series of the Nicholson-Bailey model is shown in Fig. 1. Both populations grow at low densities, but at large host densities the parasitoid begins to overexploit the host. This leads to a crash in the host population, followed by a crash of the parasitoids. These cycles of overexploitation and crashes result in an unstable interaction with both populations exhibiting diverging oscillations. Interestingly, these diverging oscillations have been recapitulated in several laboratory studies of specific host-parasitoid species providing some model validation, at least in controlled lab settings. Before discussing generalizations to the Nicholson-Bailey model, we briefly review mathematical approaches used for dissecting stability in discrete-time models. 


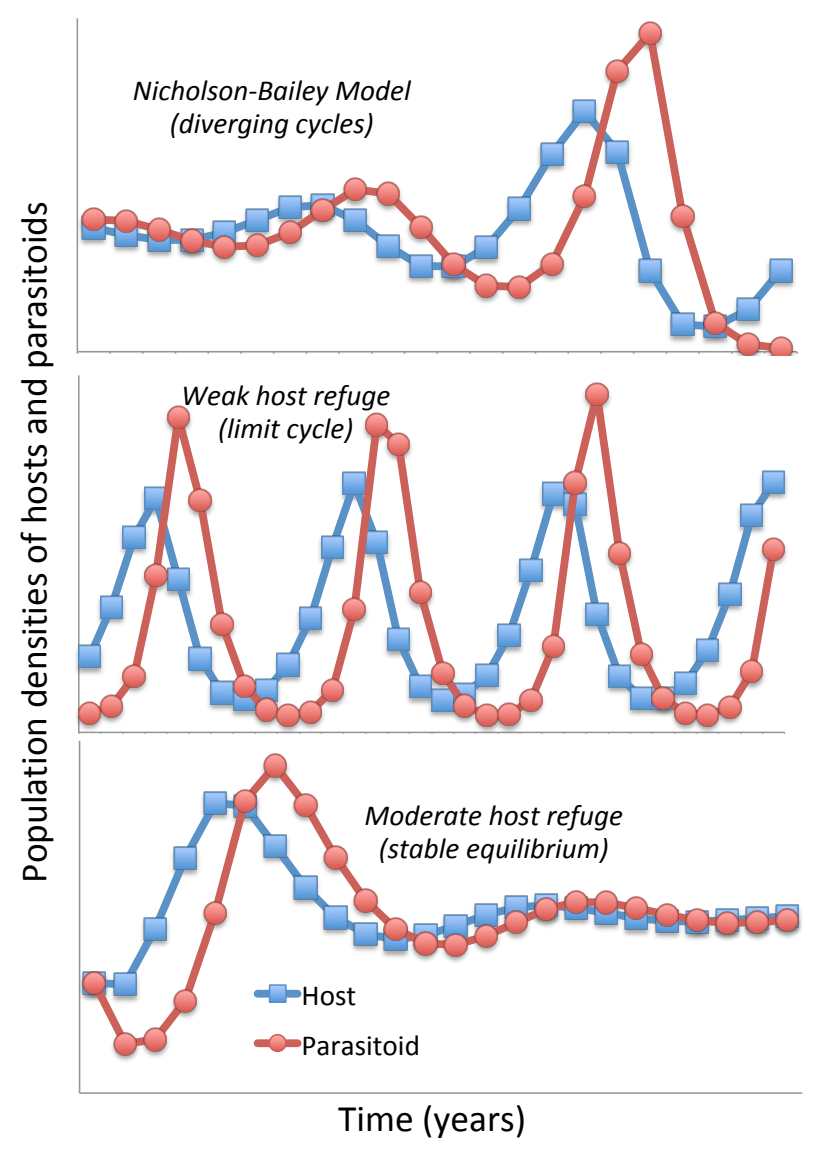

Figure 1: A typical host-parasitoid population time series for the Nicholson-Bailey model (top), model with weak host refuge ( $\mu=0.05$ in (16); middle) and moderate host refuge ( $\mu=0.20$ in (16); bottom). Host reproduction rate assumed to be $R=2$.

\section{General Stability Analysis}

Given a model of the form (1), one is typically interested in knowing if the model can support a stable host-parasitoid interaction, and if yes, then in what parameter regimes. While numerically simulating the model for a few test parameters can provide the first answers, a systematic quantification of stability regions proceeds along the following steps:

\section{Step 1: Determining the equilibrium point}

The model's equilibrium or fixed points are the populations densities that remain constant across years. Substituting $P_{t+1}=P_{t}=P^{*}$ and $H_{t+1}=H_{t}=H^{*}$ in (1) shows that the non-trivial equilibrium is the solution to the following equations

$$
1=R f\left(H^{*}, P^{*}\right), P^{*}=k(R-1) H^{*},
$$


where $H^{*}$ and $P^{*}$ denote the host and parasitoid densities at equilibrium, respectively. Solving these equations for the Nicholson-Bailey model yields a single non-trivial equilibrium point

$$
H^{*}=\frac{\ln (R)}{(R-1) k c T}, P^{*}=\frac{\ln (R)}{c T} .
$$

Note that the host equilibrium levels decrease, while the parasitoid equilibrium levels increase, with increasing host growth rate $R$.

\section{Step 2: Linearizing around the equilibrium}

Real populations are never in equilibrium as fluctuations in model parameters and unmodeled interactions constantly perturb the system out of equilibrium. If these perturbations transiently decay and the populations return to equilibrium, then the equilibrium point is said to be stable. Alternatively, if perturbations amplify, and the populations increasingly deviate from the equilibrium, then the equilibrium point is said to be unstable. Considering small perturbations $h_{t}$ and $p_{t}$

$$
h_{t}=H_{t}-H^{*}+h_{t}, \quad p_{t}=P_{t}-P^{*},
$$

and linearizing model nonlinearities in (1) around the equilibrium, results in the following linear discrete-time system

$$
\left[\begin{array}{l}
h_{t+1} \\
p_{t+1}
\end{array}\right]=A\left[\begin{array}{l}
h_{t} \\
p_{t}
\end{array}\right], A=\left[\begin{array}{ll}
a_{00} & a_{01} \\
a_{10} & a_{11}
\end{array}\right]
$$

where the entries of the $A$ matrix are given by

$$
\begin{aligned}
& a_{00}=1+\left.R H^{*} \frac{\partial f\left(H_{t}, P_{t}\right)}{\partial H_{t}}\right|_{H_{t}=H^{*}, P_{t}=P^{*}} \\
& a_{01}=\left.R H^{*} \frac{\partial f\left(H_{t}, P_{t}\right)}{\partial P_{t}}\right|_{H_{t}=H^{*}, P_{t}=P^{*}} \\
& a_{10}=k(R-1)-\left.k R H^{*} \frac{\partial f\left(H_{t}, P_{t}\right)}{\partial H_{t}}\right|_{H_{t}=H^{*}, P_{t}=P^{*}} \\
& a_{11}=-\left.k R H^{*} \frac{\partial f\left(H_{t}, P_{t}\right)}{\partial P_{t}}\right|_{H_{t}=H^{*}, P_{t}=P^{*}} .
\end{aligned}
$$

Here $\left.\frac{\partial f\left(H_{t}, P_{t}\right)}{\partial H_{t}}\right|_{H_{t}=H^{*}, P_{t}=P^{*}}$ represents the partial derivative of the escape response with respect to the host density evaluated at the equilibrium point. The matrix $A$ is mathematically referred to as the Jacobian matrix and its eigenvalues are intricately linked to the stability of the equilibrium. 


\section{Step 3: Checking for stability}

The equilibrium point is stable if the following three conditions hold

$$
1-\operatorname{tr}(A)+\operatorname{det}(A)>0,1+\operatorname{tr}(A)+\operatorname{det}(A)>0,1-\operatorname{det}(A)>0
$$

where the trace and determinant of the $A$ matrix are

$$
\operatorname{tr}(A)=a_{00}+a_{11}, \operatorname{det}(A)=a_{00} a_{11}-a_{01} a_{10},
$$

respectively [5]. It turns out that in many cases these stability conditions can be further simplified. For example, if the escape response $f\left(P_{t}\right)$ only depends on parasitoid density, then the first two conditions in (8) always hold, and stability is completely determined by the third inequality $1-\operatorname{det}(A)>0$. If $1-\operatorname{det}(A)=0$ then the equilibrium point is said to neutrally stable (on the edge of stability and instability), and both host and parasitoid populations cycle with a period of 6 or higher. If $1-\operatorname{det}(A)>0$ then the equilibrium is unstable, and populations either show diverging oscillations that grow unboundedly (as in the Nicholson-Bailey model), or they settle into bounded population cycles (i.e., a stable limit cycle).

Interestingly, the inequality $1-\operatorname{det}(A)>0$ can be rewritten in a different form

$$
\frac{d H^{*}}{d R}>0
$$

which leads to a simple, yet powerful stability condition - the model's equilibrium is stable, if and only if, the host equilibrium density increases with increasing host growth rate $R$ [6]. Recall that $H^{*}$ in the Nicholson-Bailey model is decreasing with $R$, and its instability is reflective of this simplified stability criterion. Using the fact that $P^{*}=k(R-1) H^{*}$, the above condition on $H^{*}$ can also be written in terms of the parasitoid equilibrium density

$$
\frac{R}{P^{*}} \frac{d P^{*}}{d R}>\frac{R}{R-1},
$$

revealing that stability requires parasitoid densities to increase sufficiently fast with increasing host growth rate. It is important to emphasize that conditions (10) and (11) are only to be used for a host-independent escape response $f\left(P_{t}\right)$. When $f\left(H_{t}, P_{t}\right)$ depends on both populations, the stability conditions (8) can be graphically represented in terms of two biologically-relevant quantities:

$$
\frac{d H^{*}}{d R} \text { and } \frac{d f\left(H^{*}, P^{*}\right)}{d H^{*}},
$$

where the latter denotes the sensitivity of the escape response to the host density. Fig. 2 shows that stability arises in a narrow band, and is more likely to occur when the escape response is a decreasing function of the host density, rather than an increasing function. 


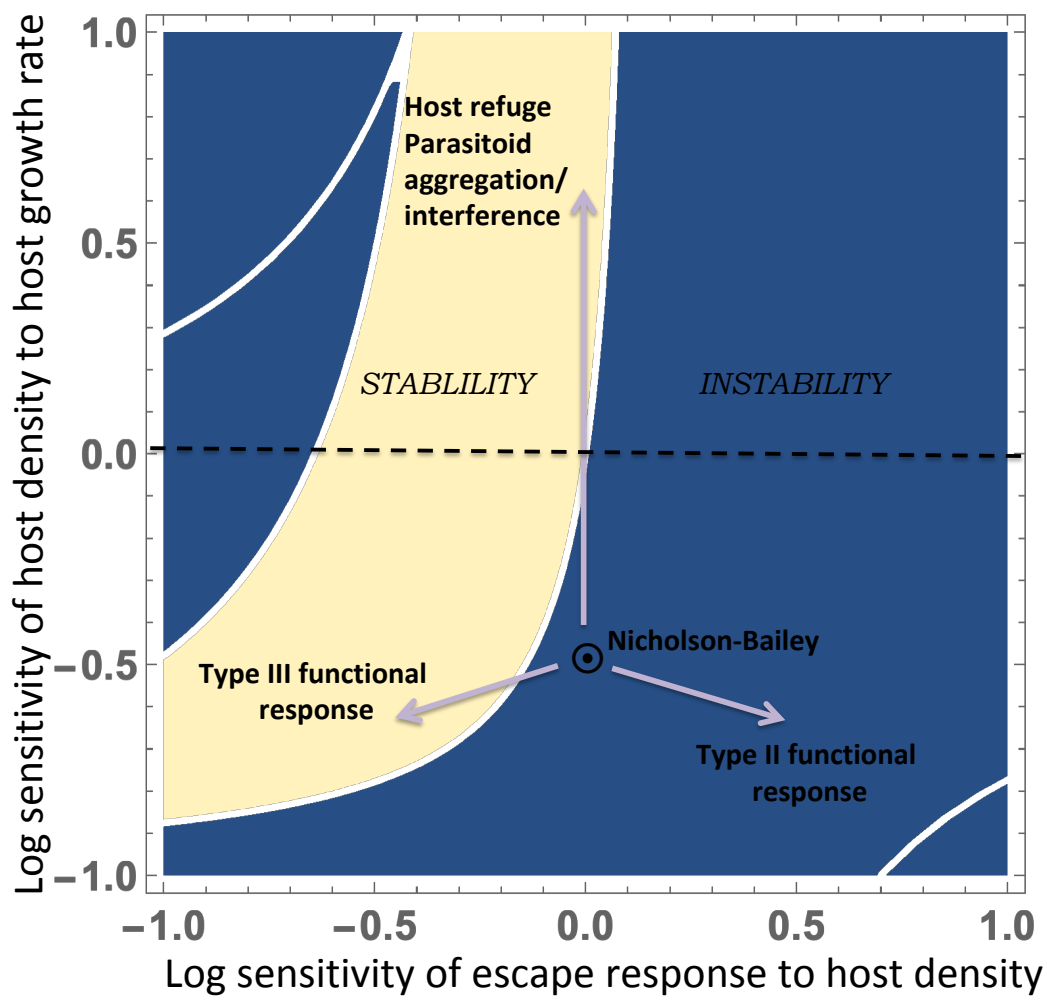

Figure 2: Stability regions for the host-parasitoid model (1) in terms of the sensitivity of the host density to the host growth rate $d H^{*} / d R$, and the sensitivity of the escape response to the host density $d f / d H^{*}$. The Nicholson-Bailey model corresponds to $d H^{*} / d R<0$ and $d f / d H^{*}=0$ and is unstable. Stability arises in two orthogonal ways: 1) an increase in $d H^{*} / d R$ to make it positive, which happens with parasitoid interference/aggregation or host refuge; 2) a decrease in $d f / d H^{*}$ which happens with a Type III functional response in the parasitoid attack rate. For this figure, the host growth rate is assumed to $R=2$, and the stability region shrinks with increasing $R$.

\section{Expanding the Nicholson-Bailey Model}

We next discuss expansions of the Nicholson-Bailey model emphasizing the stabilizing and destabilizing effects of different mechanisms. 


\section{Host and parasitoid-dependent attack rates}

The Nicholson-Bailey model assumes that parasitoids search and attack hosts with a constant rate $c$, implying a Type I functional response. This assumption is relaxed by considering a Type II functional response. Prior studies have implemented it by modifying the attack rate in (3) to

$$
c=\frac{c_{1}}{1+c_{1} T_{h} R H_{t}}
$$

where $c_{1}>0$ is the attack rate at small host densities, and $T_{h}$ is the handling time [7]. With this change, the escape response is now an increasing function of $H_{t}$, and it makes the model even more unstable by moving it further away from the stability boundary in Fig 2. A Type III functional response is incorporated by setting

$$
c=\frac{c_{1}\left(R H_{t}\right)^{q}}{1+c_{1} T_{h}\left(R H_{t}\right)^{q+1}}, q \geq 1
$$

and fails to stabilize irrespective of the value of $q$ [8]. The fact that a Type III response in not stabilizing in the Nicholson-Bailey framework is surprising, since they are known to have a stabilizing effect in the continuous-time framework of Lotka-Volterra models. As discussed later on, this discrepancy arises from how the Type III response is phenomenologically introduced in (14). Indeed, modeling host mortality during the vulnerable period as a continuous-time process confirms the stabilizing properties of a Type III response, removing the discrepancy between the two frameworks.

In contrast to a host-dependent attack rate, a parasitoid-dependent attack rate is stabilizing. Consider the scenario where the attack rate is proportional to $P_{t}^{-m}, 0<$ $m<1$, and decreases with increasing $P_{t}$ due to parasitoid interference. Since the escape response only depends on $P_{t}$, stability can be discerned by simply testing for host equilibrium density increasing with growth rate. Solving for the host equilibrium and applying (10), reveals the system to be stable for

$$
\frac{R \ln (R)+1-R}{R \ln (R)}<m<1,
$$

which corresponds to $0.28<m<1$ for $R=2$, and $0.5<m<1$ for $R=5$. In the context of Fig. 2, stability arises by moving the Nicholson-Bailey model upwards via a change in the sign of $d H^{*} / d R$.

\section{Host refuge}

Host refuges can be incorporated in the Nicholson-Bailey model in two different ways: a constant host density protected from parasitism, or a constant fraction of 
hosts in the refuge. Both forms of the refuge stabilize host-parasitoid dynamics. For example, a constant host fraction $\mu$ in the refuge leads to the following model

$$
\begin{aligned}
H_{t+1} & =R H_{t}\left(\mu+(1-\mu) \exp \left(-c T P_{t}\right)\right) \\
P_{t+1} & =k(1-\mu) R H_{t}\left[1-\exp \left(-c T P_{t}\right)\right]
\end{aligned}
$$

[1]. While a weak-refuge results in bounded oscillations, a moderate-refuge stabilizes the population dynamics (Fig. 1). However, stability is again lost for a strong-refuge with both hosts and parasitoids growing unboundedly. As before, the stability regime in terms of $\mu$ can be obtained by simply checking (10).

\section{Variation in Risk}

The stability arising from a host refuge can be generalized under the concept of variation in risk. The Nicholson-Bailey model assumes that all hosts are identical in terms of their vulnerability to parasitism. Perhaps a more realistic scenario is individual hosts differing in their risk of parasitism due to genetic factors, spatial heterogeneities, or are exposed to parasitoids for different durations, and at different times. In essence, the product $c T$ in (3) represents the attack rate integrated over time, and by transforming it into a random variable we obtain

$$
\begin{aligned}
H_{t+1} & =R H_{t} \int_{x=0}^{\infty} p(x) \exp \left(-x P_{t}\right) \\
P_{t+1} & =k R H_{t}\left[1-\int_{x=0}^{\infty} p(x) \exp \left(-x P_{t}\right)\right]
\end{aligned}
$$

where $p(x)$ is the distribution of risk across hosts [6]. A key assumption in this formulation is that risk is independent of the local host density, if hosts are nonuniformly distributed in space. Assuming $p(x)$ follows a gamma distribution with mean $\bar{c}$ and coefficient of variation $C V$,

$$
\int_{x=0}^{\infty} p(x) \exp \left(-x P_{t}\right)=\frac{1}{\left(1+\bar{c} C V^{2} P_{t}\right)^{\frac{1}{c V^{2}}}},
$$

in (17), and stability analysis of this model leads to a classical result - $C V>1$ stabilizes the population dynamics irrespective of all other model parameters $(R$, $k$ and $\bar{c})[9,10,11]$. The stabilizing risk distribution is shown in Fig. 3 where a majority of hosts are at low risk, and stability arises from parasitoid attacks being skewed towards a small fraction of high-risk individuals. This stability criterion motivated several studies investigating spatial pattern of parasitism in the field, and many data sets were found to be consistent with $C V>1$ [12]. 


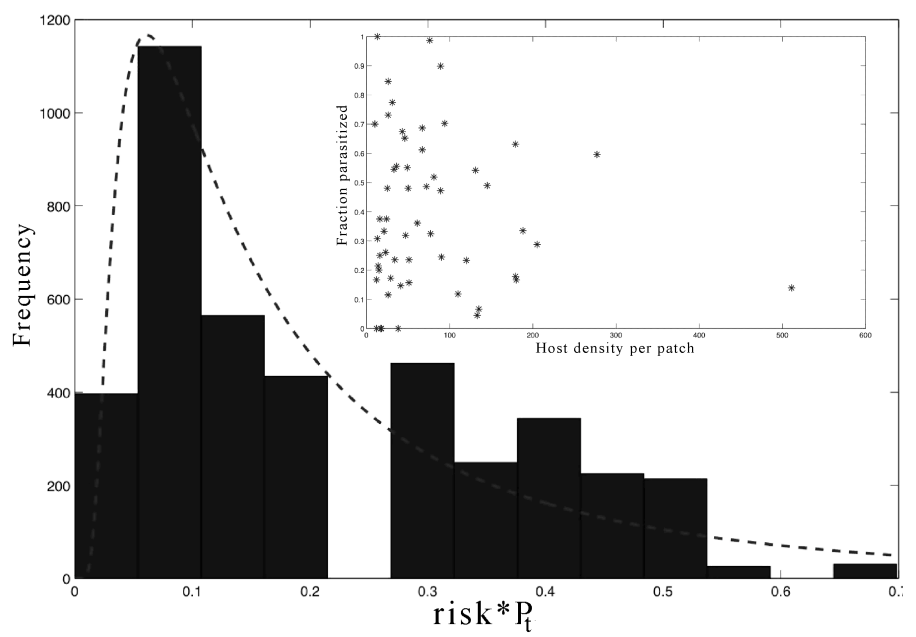

Figure 3: The inset shows fraction host parasitized vs. host density across all patches taken from [13]. The corresponding distribution of risk has a $C V$ of 1.30, and is approximated by an inverse Gaussian distribution (dashed-line). $P_{t}$ denotes the parasitoid density in year $t$.

Recent work in this direction has relaxed the assumption of a gamma-distributed risk. It turns out that if $R \approx 1$, then $C V>1$ is the necessary and sufficient condition for stability irrespective of what form $p(x)$ takes. However, for $R \gg 1$, stability requires a skewed risk distribution with the modal risk being zero (as in the gamma distribution for $C V>1$ ). We illustrate this point with the data presented in Fig. 3 where $p(x)$ is approximated by an inverse Gaussian distribution that has a non-zero mode. In spite of having of a $C V=1.30$, this risk distribution is stabilizing only for $1<R<2$. Interestingly, if the host risk follows an inverse Gaussian distribution and $R>5$, then the host-parasitoid equilibrium can never be stabilized irrespective of how high $C V$ is. In summary, for host growth rates close to one, sufficient variation in host risk $(C V>1)$ is stabilizing. In contrast, at high growth rates, the shape of the distribution for low-risk individuals is crucial in determining stability [6].

\section{Discrete-Continuous Hybrid Models}

As illustrated above, for a majority of host-parasitoid models the escape response is phenomenologically chosen or designed to recapitulate field observations. While 
these models have tremendously improved our understanding of stabilizing processes, mechanistic modeling frameworks are needed to translate insect lifestyles and behaviors into discrete-time models. For example, consider a scenario where the parasitoids have a density-dependent mortality from predation or food limitation. It is not obvious how to modify the Nicholson-Bailey model to reflect this density-dependence. For this purpose, semi-discrete or hybrid frameworks have been proposed that use ordinary differential equations to track population densities within the host vulnerable period of a given year $[14,15,16]$. The solution of the differential equations at the end of the vulnerable period predicts the population densities for next year. We discuss this semi-discrete formulation in further detail below.

Let $\tau$ denote the time within the host vulnerable stage that varies from 0 to $T$ corresponding to the start and end of the vulnerable stage. The density of parasitoids $(P)$, un-parasitized $(L)$ and parasitized host larvae $(I)$ at time $\tau$ within the vulnerable stage of year $t$, evolves according to the following differential equations

$$
\begin{aligned}
& \frac{d P(\tau, t)}{d \tau}=-c P(\tau, t) L(\tau, t)-\gamma_{P} P(\tau, t) \\
& \frac{d L(\tau, t)}{d \tau}=-c P(\tau, t) L(\tau, t)-\gamma_{L} L(\tau, t) \\
& \frac{d I(\tau, t)}{d \tau}=c P(\tau, t) L(\tau, t)-\gamma_{I} I(\tau, t),
\end{aligned}
$$

respectively. Here $c$ represents the parasitoid's attack rate, and $\gamma_{P}, \gamma_{L}, \gamma_{I}$ are the mortality rates. Solving the differential equations with initial conditions at the start of the vulnerable period $(\tau=0)$

$$
L(0, t)=R H_{t}, \quad P(0, t)=P_{t}, I(0, t)=0
$$

predicts the parasitized and unparasitized larval population at the end of the season $(\tau=T)$. This leads to a more general discrete-time model

$$
\begin{aligned}
H_{t+1} & =F\left(H_{t}, P_{t}\right) \\
P_{t+1} & =G\left(H_{t}, P_{t}\right)
\end{aligned}
$$

where update functions are obtained by setting $F\left(H_{t}, P_{t}\right)=L(T, t)$ and $G\left(H_{t}, P_{t}\right)=$ $k I(T, t)$. As expected, a constant attack rate $c$ with no mortalities $\left(\gamma_{P}=\gamma_{L}=\gamma_{I}=0\right)$ results in the Nicholson-Bailey model. 


\section{Revisiting functional responses}

A functional response can be incorporated in the semi-discrete framework by having the attack rate take the form

$$
c=\frac{c_{1} L(\tau, t)^{q}}{1+c_{1} T_{h} L(\tau, t)^{q+1}}
$$

where $q=0$ corresponds to a Type II response, and $q \geq 1$ a sigmoidal Type III response. Singh \& Nisbet 2007 show that the resulting discrete-time model is stable for $q>1$ assuming that the handling time is significantly shorter than the vulnerable stage duration $\left(T_{h} \ll T\right)$. Interestingly, the host equilibrium here is still a decreasing function of the host growth rate (as in the Nicholson-Bailey model), but a Type III response makes the fraction of hosts escaping parasitism a decreasing function of the host density (Fig. 2).

It is noteworthy to contrast the attack rate (28) with the phenomenologically constructed functional response (14). In (28), the attack rate depends on the current host density, which decreases over time due to parasitism. In contrast, the attack rate in (28) is set by the initial host density, and remains constant (and high) throughout the vulnerable period. As a consequence, for a given parasitoid density, the fraction escaping parasitism is higher for (28) as compared to (14), and this provides a stabilizing influence on the host-parasitoid dynamics. Finally, we point out that a Type I response $(q=0)$ is destabilizing in both formulations.

\section{Density-dependent host mortality}

A key mechanism known to have a stabilizing effect on the host-parasitoid populations is the density-dependent self-limitation in the host $[17,18,19]$. Densitydependent host mortality can be modeled by assuming that the death rate $\gamma_{L}=$ $c_{h} L(\tau, t)$ is proportional to the host density. For a constant parasitoid attack rate and assuming $k=1$, solving the continuous dynamics (19) gives the following discrete-time model

$$
\begin{aligned}
& H_{t+1}=L(1, t)=\frac{R H_{t} \exp \left(-c P_{t}\right)}{1+c_{h} R H_{t} \frac{1-\exp \left(-c P_{t}\right)}{c P_{t}}} \\
& P_{t+1}=I(1, t)=\frac{c P_{t}}{c_{h}} \ln \left[1+c_{h} R H_{t} \frac{1-\exp \left(-c P_{t}\right)}{c P_{t}}\right] .
\end{aligned}
$$

In the absence of parasitoids, this would lead to

$$
H_{t+1}=\frac{R H_{t}}{1+c_{h} R H_{t}} \text {. }
$$




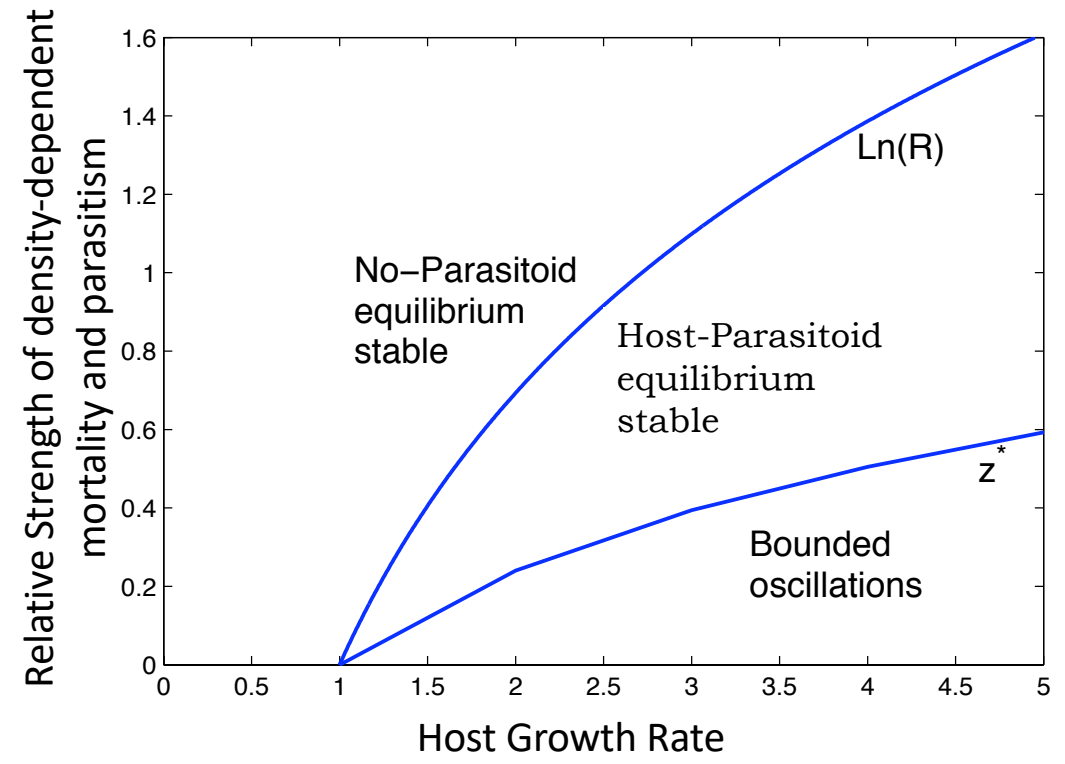

Figure 4: Stability regions for the different equilibriums in the discrete-time model (22) with density-dependent host mortality and constant parasitoid attack rate.

The above system has two non-trivial equilibrium points. The first is the noparasitoid equilibrium that is set by the strength of the host-density dependence

$$
H^{*}=\frac{R-1}{c_{h} R}, P^{*}=0,
$$

and is stable for

$$
\ln R<\frac{c_{h}}{c},
$$

a condition that precludes growth of a small parasitoid population when the hosts are at carrying capacity. Thus, sufficiently large values of $c_{h} / c$, which can be interpreted as the strength of intraspecific competition vs. parasitism, stabilize the no-parasitoid equilibrium. The second equilibrium, where both host and parasitoid are present is given by

$$
H^{*}=\left(\frac{\exp \left(\frac{c_{h}}{c}\right)-1}{1-\frac{\exp \left(\frac{c_{h}}{c}\right)}{R}}\right) \frac{c P^{*}}{c_{h} R}, \quad P^{*}=\frac{\ln R-\frac{c_{h}}{c}}{c},
$$


and is stable for

$$
z^{*}<\frac{c_{h}}{c}<\ln R
$$

where constant $z^{*}$ is an increasing function of $R$, however increasing not as fast as $\ln R$. Thus, the size of the stability region in (27) increases with $R$ (Fig. 4). As summarized in Fig. 4, for weak parasitism the no-parasitoid equilibrium is stable, moderate levels of parasitism stabilize the host-parasitoid interaction, and stability is again for lost strong parasitism with both populations exhibiting bounded oscillations.

The quantity

$$
\frac{R\left(\exp \left(\frac{c_{h}}{c}\right)-1\right)\left(\ln R-\frac{c_{h}}{c}\right)}{(R-1)\left(R-\exp \left(\frac{c_{h}}{c}\right)\right)}
$$

is of special interest to biological control, as it represents the ratio of the host equilibrium with parasitoids and without parasitoids. As one varies $c_{h} / c$ in the stability region (27), it can be used to obtain the maximum level of host depression that is consistent with a stable dynamics. This formula predicts an approximate $70 \%$ depression of hosts for growth rates in the range $R=2-10$. While the parasitoid attack rate is assumed to be constant here, it will be interesting to see how a combination of density-dependence in host mortality and parasitoid attack shapes stability, and the degree of host suppression.

\section{Density-dependent parasitoid mortality}

Singh \& Nisbet 2007 show that density-dependence in the parasitoid mortality rate modeled by taking $\gamma_{P}=c_{P} P(\tau, t)$ in (19) results in a model identical to the one obtained by assuming a gamma-distributed host risk. The only difference being that $\bar{c}$ in (18) is replaced by $c$, and $C V^{2}$ by $c_{P} / c$. As discussed earlier, stability arises in the model when $C V>1$, implying that strong density-dependent parasitoid mortality $c_{p}>c$ can stabilize the host-parasitoid interaction.

\section{Host feeding}

Recent work has used the semi-discrete framework to investigate the effect of host feeding, which refers to the tendency of some parasitoids to use the host as a food/energy source by feeding on them rather than laying an egg inside [20, 21, $22,23,24]$. In [25], the authors consider each adult parasitoid to be in one of the two states: without egg and with egg. Host feeding results in the death of 
the host with an eggless parasitoid gaining energy to produce an egg. Moreover, host parasitism by an egg-carrying parasitoid results in an infected host and an eggless parasitoid. Analysis of such simple models using the semi-discrete framework shows that while host feeding by itself cannot stability the otherwise unstable Nicholson-Bailey model equilibrium consistent with other studies [26, 27], it can have a stabilizing effect when coupled to other stabilizing mechanisms [25].
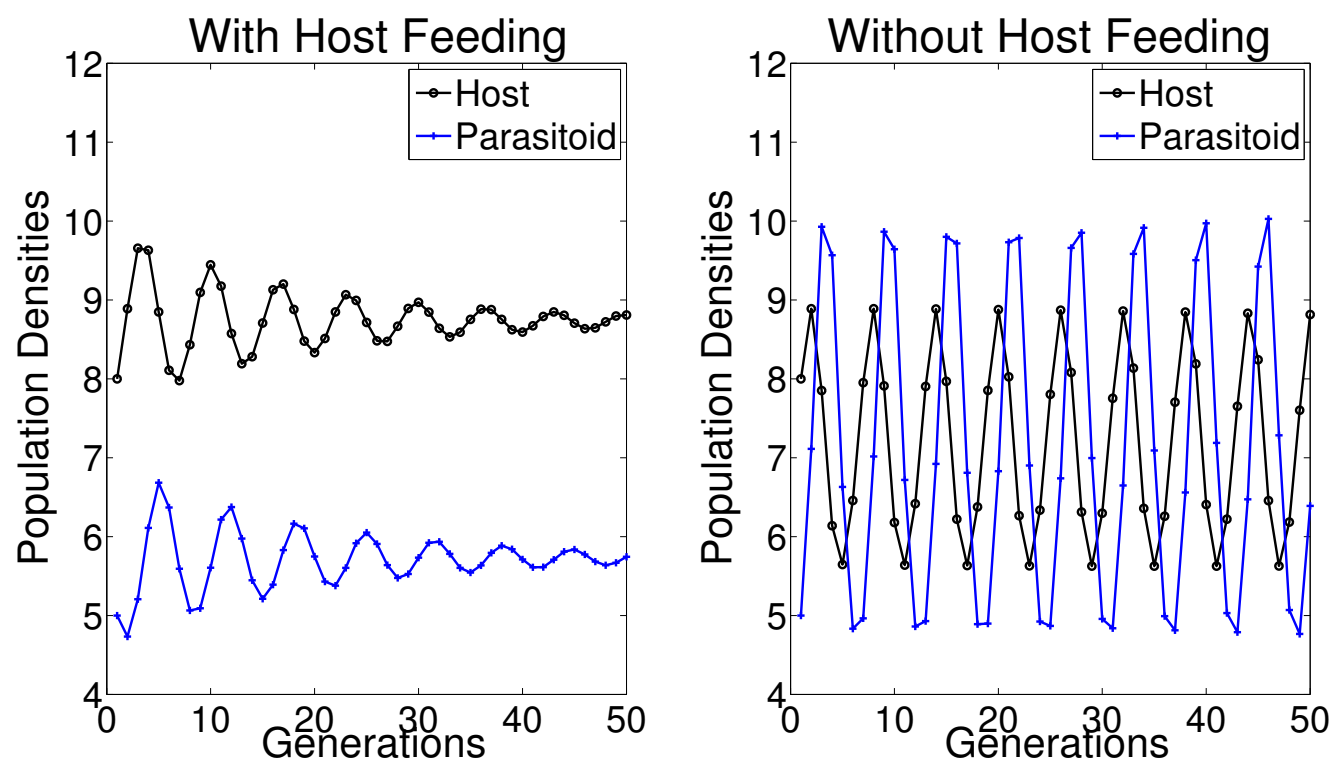

Figure 5: Coupling of host feeding with a quadratic parasitoid attack rate stabilizes an otherwise neutrally stable host-parasitoid population dynamics [25].

This point is illustrated by considering a quadratic Type-III functional response

$$
c=c_{1} L(\tau, t)
$$

with the parasitoid attack rate (for both host feeding and parasitism) increasing linearly with the larval population density. It is important to point out that such a functional response leads to a neutrally stable host-parasitoid equilibrium in the absence of host feeding [15]. Incorporation of host feeding yields the following 
continuous dynamics during the vulnerable stage

$$
\begin{aligned}
\frac{d L}{d \tau} & =-c\left(P_{0}+P_{1}\right) L^{2} \\
\frac{d I}{d \tau} & =c P_{1} L^{2} \\
\frac{d P_{0}}{d \tau} & =c\left(P_{1}-P_{0}\right) L^{2} \\
\frac{d P_{1}}{d \tau} & =-c\left(P_{1}-P_{0}\right) L^{2} .
\end{aligned}
$$

where $P_{0}$ and $P_{1}$ represent populations densities of parasitoids without and with egg, respectively [25]. Solving (29) results in the following discrete-time model

$$
\begin{aligned}
H_{t+1} & =\frac{R H_{t}}{1+c R H_{t} P_{t}} \\
P_{t+1} & =\frac{R H_{t}-H_{t+1}+\frac{P_{t}}{2}\left\{1-\exp \left[-\frac{2\left(R H_{t}-H_{t+1}\right)}{P_{t}}\right]\right\}}{2} .
\end{aligned}
$$

which exhibits a stable host-parasitoid equilibrium for all values of all $R$. Thus, inclusion of host feeding converts a neutrally stable equilibrium to a stable one (Fig. 5).

\section{References}

[1] W. W. Murdoch, C. J. Briggs, and R. M. Nisbet. Consumer-Resouse Dynamics. Princeton University Press, Princeton,NJ, 2003.

[2] M. P. Hassell. Oxford University Press, New York, 2000.

[3] W. S. C. Gurney and R. M. Nisbet. Ecological Dynamics. Oxford University Press, 1998.

[4] A.J. Nicholson and V. A. Bailey. The balance of animal populations. part 1. Proc. of Zoological Society of London, 3:551-598, 1935.

[5] S. Elaydi. An Introduction to Difference Equations. Springer, Newyork, 1996.

[6] A. Singh, W. W. Murdoch, and R. M. Nisbet. Skewed attacks, stability, and host suppression. Ecology, 90(6):1679-1686, 2009.

[7] D. J. Rogers. Random searching and incest population models. J. of Animal Ecology, 41:369-383, 1972. 
[8] M. P. Hassell and H. N. Comins. Sigmoid functional responses and population stability. Theoretical Population Biology, 14:62-66, 1978.

[9] R. M. May. Host-parasitoid systems in patchy environments: a phenomenological model. Journal of Animal Ecology, 47:833-844, 1978.

[10] A. D. Taylor. Heterogeneity in host-parasitoid interactions: 'aggregation of risk' and the ' $\mathrm{cv}^{2}>1$ rule.'. Trends in Ecology and Evolution, 8:400-405, 1993.

[11] M. P. Hassell, R. M. May, S. W. Pacala, and P. L. Chesson. The persistence of host-parasitoid associations in patchy environments. I. a general criterion. American Naturalist, 138:568-583, 1991.

[12] S. W. Pacala and M. P. Hassell. The persistence of host- parasitoid associations in patchy environments. II. evaluation of field data. American Naturalist, 138:584-605, 1991.

[13] J. D. Reeve, J. T. Cronin, and D. R. Strong. Parasitoid aggregation and the stabilization of a salt marsh host- parasitoid system. Ecology, 75:288-295, 1994.

[14] A. Singh and R. M. Nisbet. Variation in risk in single-species discrete-time models. Mathematical Biosciences and Engineering, 5:859-875, 2008.

[15] A. Singh and R. M. Nisbet. Semi-discrete host-parasitoid models. J Theor Biol, 247(4):733-742, 2007.

[16] B. K. Emerick and A. Singh. Global redistribution and local migration in semi-discrete host-parasitoid population dynamic models. bioRxiv, 2019.

[17] M. G. Neubert and M. Kot. The subcritical collapse of predator populations in discrete-time predator-prey models. Mathematical Bioscience, 110:45-66, 1992.

[18] M. P. Hassell and G. C. Varley. New inductive population model for insect parasites and its bearing on biological control. Nature, 223:1133 - 1137, 1969.

[19] R. M. May, M. P. Hassell, R. M. Anderson, and D. W. Tonkyn. Density dependence in host-parasitoid models. J. of Animal Ecology, 50:855-865, 1981.

[20] B. A. Hawkins. Patterns and Process in Host-Parasitoid Interactions. Cambridge University Press, New York, NY, 1994. 
[21] M. A. Jervis and N. A. C. Kidd. Host-feeding strategies in hymenopteran parasitoids. Biol Rev, 61(4):395-434, 1986.

[22] N. A. C. Kidd and M. A. Jeris. The effects of host-feeding behaviour on the dynamics of parasitoid-host interactions, and the implications for biological control. Res Popul Ecol, 31(2):2435-274, 1989.

[23] M. A. Jervis, B. A. Hawkin, and N. A. C. Kidd. The usefulness of destructive host-feeding parasitoids in classical biological control: theory and observation conflict. Ecological Entomology, 21(1):41-46, 1996.

[24] T. Ueno. Selective host-feeding on parasitized hosts by the parasitoid itoplectis naranyae (hymenoptera: Ichneumonidae) and its implication for biological control. Bullletin of Entomological Research, 88(4):461-466, 1998.

[25] B. K. Emerick and A. Singh. The effects of host-feeding on stability of discrete-time host-parasitoid population dynamic models. Mathematical Biosciences, 272:54-63, 2016.

[26] C. J. Briggs, R. M. Nisbet, W. W. Murdoch, T. R. Collier, and J. Metz. Dynamical effects of host-feeding. J Anim Ecol, 64(3):403-416, 1995.

[27] N. A. C. Kidd and M. A. Jervis. Host-feeding and oviposition strategies of parasitoids in relation to host stage. Res Popul Ecol, 33(1):13-28, 1991. 